\title{
Convexity adjustment for constant maturity swaps in a multi-curve framework
}

\author{
Nikolaos Karouzakis $^{1}$ (D) John Hatgioannides ${ }^{2} \cdot$ \\ Kostas Andriosopoulos ${ }^{3}$
}

Published online: 6 March 2017

(c) The Author(s) 2017. This article is published with open access at Springerlink.com

\begin{abstract}
In this paper we propose a double curving setup with distinct forward and discount curves to price constant maturity swaps (CMS). Using separate curves for discounting and forwarding, we develop a new convexity adjustment, by departing from the restrictive assumption of a flat term structure, and expand our setting to incorporate the more realistic and even challenging case of term structure tilts. We calibrate CMS spreads to market data and numerically compare our adjustments against the Black and SABR (stochastic alpha beta rho) CMS adjustments widely used in the market. Our analysis suggests that the proposed convexity adjustment is significantly larger compared to the Black and SABR adjustments and offers a consistent and robust valuation of CMS spreads across different market conditions.
\end{abstract}

Keywords Convexity adjustment - Constant maturity swaps - Multi-curve framework · Yield curve modelling $\cdot$ Money market instruments

\section{Introduction}

The recent financial crisis has led, among others, to unprecedented behavior in the money markets, which has created important discrepancies on the valuation of interest rate financial instruments. Important reference rates that used to be highly correlated and moving together for a long period of time, started to diverge from one another. A characteristic example,

\footnotetext{
$\bowtie$ Nikolaos Karouzakis

N.Karouzakis@sussex.ac.uk

John Hatgioannides

j.hatgioannides@city.ac.uk

Kostas Andriosopoulos

kandriosopoulos@escpeurope.eu

1 School of Business, Management and Economics, University of Sussex, Brighton BN1 9SL, UK

2 Cass Business School, City University London, 106 Bunhill Row, London EC1Y 8TZ, UK

3 ESCP Europe Business School, 527 Finchley Road, London NW3 7BG, UK
} 
that has been widely studied recently, is the widening of the spread between deposit rates (Libor/Euribor) and overnight index swap (OIS) rates of the same maturity. At the same time, the market started observing non-zero spreads between swap rates of the same maturity, but based on different frequencies of the underlying Libor rate, or between forward rate agreement (FRA) rates and forward rates implied by consecutive deposits. These examples indicate that financial players consider each tenor as a separate market, incorporating different credit and liquidity premia, and as such, each one of them is driven by its own dynamics.

Such discrepancies have, above all, questioned the methodology used to bootstrap the yield curve, which has created a layer of uncertainty on the methods used to price and hedge interest rate financial instruments. There are three main issues associated with the pre-crisis approach, which make it inconsistent. First, the information incorporated into the basis spreads is not taken into account. Second, using a single yield curve does not allow us to consider the different dynamics introduced by each underlying rate tenor, making hedging and pricing of interest rate derivatives less stable. Finally, the no-arbitrage assumption indicates that a unique discounting curve needs to be used, regardless of the number of the underlying tenors.

In order for market participants to comply with the mentioned market features, they started building a separate forward curve for each given tenor, so that future cash flows are generated using the appropriate curve associated with the underlying rate tenor. At the same time, a single and unique discounting curve had to be used, in order to calculate the present value of contract's future payments. This led financial players to start using the OIS swap curve, rather than the Libor curve, for the construction of a riskless term structure. The reason behind their choice was mainly twofold. First, OIS is believed to contain very little credit and liquidity risk premia compared to Libor rates. Second, the fact that most trades in the interest rate market are (mainly cash) collateralized makes the funding cost for a financial institution no longer equal to the Libor rate, but to the collateral rate instead. For that reason, the Libor rate that was widely used as a proxy for the risk-free (discounting) rate, is now replaced by the collateral rate, which is assumed to coincide with the overnight rate (i.e. fed fund rate for USD, Eonia for EUR, etc.).

The literature on the valuation of interest rate derivatives based on separate curves, for generating future rates and for discounting, is growing rapidly. Previous contributions focus on the valuation of cross currency (basis) swaps (see, Boenkost and Schmidt 2005; Kijima et al. 2009; Fujii et al. 2010; Henrard 2010). Henrard (2007b), is the first to apply this methodology to the single currency case, whereas Bianchetti (2010) is the first to deal with the post-crisis situation. Furthermore, Ametrano and Bianchetti (2009), Chibane and Sheldon (2009) and Morini (2009), develop new methodologies for bootstrapping multiple interest rate yield curves. On the other hand, many contributions focus on extending pricing models under the multi-curve framework. Kijima et al. (2009), apply the methodology to study two short rate models, the Vasicek model and the quadratic Gaussian model, and use them for the valuation of bond options and swaptions. Mercurio (2009, 2010) and Grbac et al. (2015) extend the libor market model (LMM) to be compatible with the multi-curve practice and price caplets and swaptions, while more recently, Pallavicini and Tarenghi (2010), Crépey et al. (2012), Moreni and Pallavicini (2014) and Cuchiero et al. (2016) extend the classical HeathJarrow-Morton (HJM) framework to incorporate multiple curves in order to price interest rate products such as forward starting interest rate swaps (IRS), plain vanilla European swaptions and CMS spread options. Finally, important contributions include Crépey et al. (2015) who develop a Levy-based HJM model for credit value adjustment (CVA) and Fanelli (2016) who develop a defaultable HJM model for pricing basis swaps in a multi-curve setup.

In this paper, we follow the approach described in Mercurio (2010) and Pallavicini and Tarenghi (2010) to price a CMS. A CMS exchanges a swap rate with a fixed time to maturity 
against fixed or floating. In a common CMS, one would swap a quarterly (e.g. 3-month Libor) or semi-annual rate against a 5 or 10 -year swap rate. Whereas a regular floating rate (e.g. 6-month Libor) contains information about short-term interest rates, a CMS rate (e.g. 10-year swap rate) contains information about the overall level of the yield curve. This makes CMS a popular instrument among investors and portfolio managers. It gives investors the ability to place bets on the shape of the yield curve over time. Generally, a constant maturity payer will benefit from a flattening or inversion of the yield curve and is exposed to the risk of the yield curve steepening. It also helps portfolio managers to hedge a floating rate debt without introducing duration risk from the hedging instrument.

The mix of short and long term rates in the structure of the CMS makes its value depend on the shape of the yield curve. Standard approaches for its valuation involve the calculation of a convexity adjustment. Such convexity adjustment cannot be computed exactly, so previous literature uses either adhoc approximations or utilising unrealistic assumptions. A common assumption used in the relevant literature is that the term structure of interest rates is flat and only parallel shifts are allowed.

There are two main avenues towards pricing a CMS. In the first, one sets up a term structure model and uses some approximation method to compute the expected swap rate, under the forward measure. More specifically, Lu and Neftci (2003) follow this direction and work with two or more forward rates jointly. Using the forward libor model, they price a CMS swap and compare its empirical performance with the standard convexity adjustment proposed by Hagan (2005). They find that the convexity adjustment overestimates CMS swap rates. Similarly, Henrard (2007a) uses one-factor LMM and HJM models to approximate CMS swaps, while Brigo and Mercurio (2006) use a two-factor Gaussian short rate model (G2++ model) to model bond prices associated with CMS products. Finally, in a recent work, Wu and Chen (2010) price different CMS-type interest rate derivatives within the LMM framework. They present a new approach for finding the approximate distribution of a CMS under the forward martingale measure.

In the second direction, one uses replication arguments and the problem is formulated under the swap measure. The price is based on the implied swaption volatilities which play the role of the distribution of swap rates. For the replication procedure, the change from the forward to the swap measure is needed and the Radon-Nikodym derivatives need to be approximated. Pelsser (2003) is the first to show that the convexity adjustment can be interpreted as the side effect of a change of numeraire. He approximates the measure change by proposing a linearization of the swap rate and obtains analytical solutions to the CMS price. Hagan (2005) obtains closed-form formulae for the pricing of CMS swaps and options by relating them to the swaption market via a static replication approach. Finally, Mercurio and Pallavicini (2006) use a strike extrapolation to statically replicate CMS swap/options by modelling implied volatilities of European swaptions using the SABR model of Hagan et al. (2002). Finally, in a recent work, Zheng and Kuen Kwok (2011) propose a generalised static replication approach to hedge exotic swap contracts and annuity options using different swaptions.

The main problem with previous contributions is that the yield curve is assumed to be flat and only parallel shifts are allowed. However, in a swap where one pays Libor plus spread and receives a 10-year CMS rate, the structure is mainly sensitive to the slope of the interest rate yield curve and is almost immunised against any parallel shift. In this paper, following Hagan (2005), we apply the commonly used convexity adjustment in a new framework of double curving. We then develop a new convexity adjustment, by departing from the restricting assumption that the term structure is flat, and we allow for a tilt. Using market data for Euro money market instruments (Eonia, Euribor), CMS spreads and swaption volatilities, we find 
out that the new convexity adjustment is significantly larger than the one commonly made in the literature. We finally compare our approach with the SABR CMS adjustment, introduced by Mercurio and Pallavicini $(2005,2006)$, which is widely used in the market, and we find that our approach provides a better fit to the market's CMS spread prices.

The remaining of this paper is organised as follows. Section 2 presents the valuation framework for the main instruments (FRA, IRS, CMS) considered. Section 3 shows the main result of our work, that is a new convexity adjustment that takes into account the tilt in the term structure, under a double curving framework. Section 4 briefly depicts the smile-consistent convexity adjustment using SABR model. Sections 5 and 6 present the market data that have been used and describe numerical calculations. Finally, Sect. 7 concludes.

\section{The valuation framework}

This section introduces the definitions of basic instruments under the muti-curve environment. It mostly follows the works of Brigo and Mercurio (2006) and Mercurio (2009).

We introduce two curves, one for the discounting process, say curve ' $d$ ', and one for the forwarding, say curve ' $f$ '. Forward rates can be defined for both curves. Let us take today being time zero and consider a tenor structure $\left\{T_{i}\right\}_{i=0, \ldots, n}$, with $T_{i}<T_{i+1}$. Let $\delta_{i}=T_{i+1}-T_{i}$ be the accrual factor for the time interval $\left[T_{i}, T_{i+1}\right]$. Within this structure, for each curve, the time- $t$ (with $t \leq T_{0}$ ) value of forward rates is defined by,

$$
F_{d}\left(t ; T_{i-1}, T_{i}\right)=\frac{1}{\delta_{i}}\left[\frac{P_{d}\left(t, T_{i-1}\right)}{P_{d}\left(t, T_{i}\right)}-1\right]
$$

where $P_{d}\left(t, T_{i}\right)$, with $i=1, \ldots, n$, denoting the time- $t$ price of the $T_{i}$-maturity discount bond. Furthermore, we denote by $Q_{d}^{T_{i}}$ the $T_{i}$-forward probability measure associated with the numeraire $P_{d}\left(t, T_{i}\right)$, and by $\mathbb{E}_{d}^{T_{i}}$ the related expectation. We assume a given single discount curve for use in the calculation of all net present values (NPVs), i.e., for discounting all future cash flows. This curve is assumed to be the OIS zero-coupon curve, stripped from market OIS swap rates and is defined for every possible maturity $T_{i}$. All pricing measures we will consider are those associated with the OIS discount curve ' $d$ '. Following Mercurio (2010), we adopt the standard definition for the FRA rate.

Definition 1 Consider times $t, T_{1}, T_{2}$, with $t \leq T_{1}<T_{2}$. The time- $t$ FRA rate $F R A\left(t ; T_{1}, T_{2}\right)$ is defined as the fixed rate to be exchanged at time $T_{2}$ for the Libor rate $L\left(T_{1}, T_{2}\right)$, so that the swap has zero value at time $t$.

By no-arbitrage pricing we get,

$$
F R A\left(t ; T_{1}, T_{2}\right)=\mathbb{E}^{Q_{d}^{T_{2}}}\left[L\left(T_{1}, T_{2}\right) \mid \mathscr{F}_{t}\right]
$$

where $Q_{d}^{T_{2}}$ denoting the $T_{2}$-forward measure associated with the numeraire $P_{d}\left(t, T_{2}\right), \mathbb{E} Q_{d}^{T_{2}}$ the related expectation and $\mathscr{F}_{t}$ the 'information' available in the market at time $t$.

Proposition 1 Any simple compounded forward rate spanning a time interval ending in $T_{i}$, is a martingale under the $T_{i}$-forward measure, $F\left(u ; T_{i-1}, T_{i}\right)=\mathbb{E}_{d}^{T_{i}}\left[F\left(t ; T_{i-1}, T_{i}\right) \mid \mathscr{F}_{u}\right]$, for $0 \leq u \leq t \leq T_{i-1}<T_{i}$.

Following Bianchetti (2010) and Mercurio (2010), working under the single-curve framework, where the forward and discount curves coincide $(f \equiv d)$, from proposition 1 , the 
forward rate $F_{f}\left(t ; T_{1}, T_{2}\right)$ is by construction a martingale under $\mathbb{Q}_{d}^{T_{2}}$,

$$
F_{f}\left(t ; T_{1}, T_{2}\right)=\frac{1}{T_{2}-T_{1}}\left[\frac{P_{d}\left(t, T_{1}\right)}{P_{d}\left(t, T_{2}\right)}-1\right]=\mathbb{E}^{Q_{d}^{T_{2}}}\left[L\left(T_{1}, T_{2}\right) \mid \mathscr{F}_{t}\right]
$$

where $L\left(T_{1}, T_{2}\right)$ is the spot Libor rate defined by the usual no-arbitrage relationship between Libor rates and zero coupon bond prices, which holds for non-defaultable counterparties and instruments with no liquidity risk,

$$
L\left(T_{1}, T_{2}\right)=\frac{1}{T_{2}-T_{1}}\left[\frac{1}{P_{d}\left(T_{1}, T_{2}\right)}-1\right]=F_{f}\left(T_{1} ; T_{1}, T_{2}\right)
$$

Based on that, we can conclude that the FRA rate $F R A\left(t ; T_{1}, T_{2}\right)$ coincides with the forward Libor rate, $F R A\left(t ; T_{1}, T_{2}\right)=F_{f}\left(t ; T_{1}, T_{2}\right)$.

In the multi-curve framework, however, Eq. (4) does not hold. The forward rate $F_{f}\left(t ; T_{1}, T_{2}\right)$ is not a martingale under the forward measure $\mathbb{Q}_{d}^{T_{2}}$, and the FRA rate is different from the forward rate, $\operatorname{FRA}\left(t ; T_{1}, T_{2}\right) \neq F_{f}\left(t ; T_{1}, T_{2}\right)$. Therefore, the present value of a future Libor rate is no longer obtained by discounting the corresponding forward rate, but by discounting the corresponding FRA rate. According to Mercurio (2010), the FRA rate is the natural generalization of a forward rate to the multi-curve case. This has a straightforward implication, when it comes to the valuation of Interest Rate Swaps.

\subsection{Interest rate swap}

We show how to evaluate an IRS under the multi-curve framework. For simplicity, we assume that IRS tenors for fixed and floating legs are the same. The time- $t$ value (with $t \leq T_{0}$ ) of the floating leg payoff is calculated by taking the discounted expectation under the forward measure $\mathbb{Q}_{d}^{T_{i+1}}$,

$$
\delta_{i} P_{d}\left(t, T_{i+1}\right) \mathbb{E}^{Q_{d}^{T_{i+1}}}\left[L\left(T_{i}, T_{i+1}\right) \mid \mathscr{F}_{t}\right]
$$

Using Eq. (2), the present value of the swap's floating leg is given as,

$$
\sum_{i=0}^{n-1} \delta_{i} P_{d}\left(t, T_{i+1}\right) F R A\left(t ; T_{i}, T_{i+1}\right)
$$

Similarly, the value of the swap's fixed leg is given by the present value of the fixed coupon payments, K, paid on the fixed legs' dates as,

$$
K \sum_{i=0}^{n-1} \delta_{i} P_{d}\left(t, T_{i+1}\right)
$$

Thus, the time- $t$ value of the IRS to the fixed rate payer is given by,

$$
\operatorname{IRS}\left(t, K ; T_{i}\right)=\sum_{i=0}^{n-1} \delta_{i} P_{d}\left(t, T_{i+1}\right) F R A\left(t ; T_{i}, T_{i+1}\right)-K \sum_{i=0}^{n-1} \delta_{i} P_{d}\left(t, T_{i+1}\right)
$$

It follows that the 'fair' forward swap rate that equates the two legs at time $t \leq T_{0}$ is,

$$
S\left(t ; T_{0}, T_{n}\right)=\frac{\sum_{i=0}^{n-1} \delta_{i} P_{d}\left(t, T_{i+1}\right) F R A\left(t ; T_{i}, T_{i+1}\right)}{\sum_{i=0}^{n-1} \delta_{i} P_{d}\left(t, T_{i+1}\right)}
$$

This is the forward swap rate of an IRS, where cash flows are generated through curve ' $f$ ' and discounted with curve ' $d$ '. 


\subsection{Constant maturity swap}

A constant maturity swap contract, is a swap where one of the legs pays (receives) periodically a swap rate with a fixed time to maturity, $\mathrm{c}$, while the other leg receives (pays) either fixed or floating. More commonly, one term is set to a short term floating index such as the 3-month Libor rate, while the other leg is set to a long term fixed rate such as the 10-year swap rate.

Let $\left\{t_{i, j}\right\}_{j=0, \ldots, c}$, be a set of reset dates, associated with times $T_{i}, i=0, \ldots, n$, with $t_{i, 0} \in\left[T_{i}, T_{i+1}\right]$ and $t_{i, j}-t_{i, j-1}=\tau$, with $j=1, \ldots, c$. We suppose that $\tau=6$ months and assume for simplicity that $t_{i, 0}=T_{i}$, and we will set $\Delta=\delta / \tau$, with $\Delta$ need not be integral. The forward swap rate of the $i^{t h}$ IRS (at time determined by $t_{i, j}$ ), at time $t \leq t_{i, 0}$ denoted as, $S_{t}^{t_{i, j}} \equiv S\left(t, t_{i, 0}, t_{i, c}\right)$, is given by,

$$
S_{t}^{t_{i, j}}=\frac{\sum_{j=0}^{c-1} \tau P_{d}\left(t, t_{i, j+1}\right) F R A\left(t ; t_{i, j}, t_{i, j+1}\right)}{\sum_{j=0}^{c-1} \tau P_{d}\left(t, t_{i, j+1}\right)}
$$

where $P_{d}\left(t, t_{i, j}\right)$ denoting the time- $t$ price of the $t_{i, j}$-maturity discount bond. Furthermore, we denote by $Q_{d}^{t_{i, j}}$ the $t_{i, j}$-forward swap probability measure associated with the numeraire $P_{t}^{t_{i, j}}=\sum_{j=1}^{c} \tau P_{d}\left(t, t_{i, j}\right)$, and by $\mathbb{E}_{d}^{Q_{i, j}^{t_{i}}}$ the related expectation.

Consider now, a $c$-year CMS starting at $T_{0}$ with payment dates $T_{i}, i=1, \ldots, n$. At each payment date $T_{i}$, one party pays (receives) $\left[\delta L_{T_{i-1}}\left(T_{i-1}, T_{i}\right)+\delta R^{t}\right]$ to its counterparty and receives (pays) $\left[\delta S_{T_{i-1}}\left(t_{i-1, j-1}, t_{i-1, j}\right)\right]$ from its counterparty, where, $L_{T_{i-1}}\left(T_{i-1}, T_{i}\right)$ denotes the $\delta$-month (e.g. 3-month) spot Libor rate resetting at $T_{i-1}$ and applied to a $\delta$ period $\left[T_{i-1}, T_{i}\right], S_{T_{i-1}}\left(t_{i-1, j-1}, t_{i-1, j}\right)$, with $j=1, \ldots, c$, is the $c$-year spot swap rate, resetting (every $\tau=6$-months) at $T_{i-1}\left(=t_{i-1,0}\right)$ and applied to a $\delta$ period $\left[T_{i-1}, T_{i}\right]$ and $R^{t}$, is the CMS premium (spread), a constant chosen so that the cost of the instrument at time $t$, when the contract is initiated, is zero. For simplicity, we write $S_{T_{i-1}}^{t_{i-1}}$ for $S_{T_{i-1}}\left(t_{i-1, j-1}, t_{i-1, j}\right)$ and $L_{T_{i-1}}^{T_{i}}$ for $L_{T_{i-1}}\left(T_{i-1}, T_{i}\right)$. At time $T_{i}$, the CMS pays cashflow $c_{i}$ as,

$$
c_{i}=\delta\left[S_{T_{i-1}}^{t_{i-1, j}}-L_{T_{i-1}}^{T_{i}}-R^{t}\right]
$$

where we suppose that the counterparty pays floating (i.e. Libor + spread) and receives fixed (i.e. the swap rate). The time- $t$ value, with $t \leq T_{i-1}$, of the CMS can be obtained by taking the discounted expectation $\mathbb{E} Q_{d}^{T_{i}}$ under the forward measure $\mathbb{Q}_{d}^{T_{i}}$ corresponding to the numeraire $P_{t}^{T_{i}}=P_{d}\left(t, T_{i}\right)$, as,

$$
\begin{aligned}
V_{t}^{T_{i}} & =P_{t}^{T_{i}} \delta \mathbb{E}^{Q_{d}^{T_{i}}}\left[S_{T_{i-1}}^{t_{i-1, j}}-L_{T_{i-1}}^{T_{i}}-R^{t}\right] \\
& =P_{t}^{T_{i}} \delta\left(\mathbb{E} Q_{d}^{T_{i}}\left[S_{T_{i-1}}^{t_{i-1, j}}\right]-F R A\left(t ; T_{i-1}, T_{i}\right)-R^{t}\right)
\end{aligned}
$$

where, for the FRA, we follow Eq. (2).

At this point it is important to emphasize the fact that, naturally, the expectation used to calculate the above payoff, is associated with the payment dates $T_{i}$. However, under the forward measure, $\mathbb{Q}_{d}^{T_{i}}$, the swap rate, $S_{T_{i-1}}^{t_{i-1}}$, is not a martingale. The convexity adjustment arises since the expected payoff is calculated in a world which is forward risk neutral with respect to a zero coupon bond. In that world, the expected underlying swap rate (upon which the payoff is based), does not equal the forward swap rate. The convexity is just the difference between the expected swap rate and the forward swap rate. 
When we consider pricing CMS-type derivatives, it is convenient to compute the expectation of the future CMS rates under the forward measure, that is associated with the payment dates. However, the natural martingale measure of the CMS rate is the underlying forward swap measure. Convexity correction arises when one computes the expected value of the CMS rate under the forward measure that differs from the natural swap measure with the underlying forward swap measure as numeraire.

\section{Convexity adjustment}

Following Pelsser (2003), we define the convexity adjustment as the difference in expectation of some quantity (i.e., swap rate) when the expectations are computed under two different measures (i.e., forward and swap measures). Therefore, expectation $\mathbb{E}^{Q_{d}^{T_{i}}}\left[S_{T_{i-1}}^{t_{i-1, j}}\right]$ can be written as an expectation which is a martingale under its measure plus an adjustment. This means that the convexity adjustment is given as the difference in expectation (under the forward measure and the forward swap measure) of the forward swap rate, as,

$$
C A_{t}^{t_{i-1, j}}=\mathbb{E}^{Q_{d}^{T_{i}}}\left[S_{T_{i-1}}^{t_{i-1, j}}\right]-\mathbb{E}^{Q_{d}^{t_{i, j}}}\left[S_{T_{i-1}^{t_{i-1, j}}}\right]
$$

where under the forward swap measure $\mathbb{Q}_{d}^{t_{i, j}}$, corresponding to the numeraire $P_{t}^{t_{i, j}}=$ $\sum_{j=1}^{c} \tau P_{d}\left(t, t_{i, j}\right)$, the forward swap rate, $S_{T_{i-1}}^{t_{i-1, j}}$, is a martingale.

Assuming that the convexity adjustment $C A_{t}^{t_{i-1, j}}$ is known, the current value of the CMS is given from Eqs. (12) and (13) by,

$$
\begin{aligned}
V_{t}^{T_{i}} & =P_{t}^{T_{i}} \delta\left(\mathbb{E}^{Q_{d}^{t_{i, j}}}\left[S_{T_{i-1}}^{t_{i-1, j}}\right]+C A_{t}^{t_{i-1, j}}-\operatorname{FRA}\left(t ; T_{i-1}, T_{i}\right)-R^{t}\right) \\
& =P_{t}^{T_{i}} \delta\left(S_{t}^{t_{i-1, j}}+C A_{t}^{t_{i-1, j}}-F R A\left(t ; T_{i-1}, T_{i}\right)-R^{t}\right)
\end{aligned}
$$

where we have used that $S_{T_{i-1} t_{i-1, j}}$ is a martingale under the forward swap measure $\mathbb{Q}_{d}^{t_{i, j}}$ and the present value of the CMS is given by,

$$
P V_{t}=\sum_{i=1}^{n} V_{t}^{T_{i}}=\sum_{i=1}^{n} P_{t}^{T_{i}} \delta\left[S_{t}^{t_{i-1, j}}+C A_{t}^{t_{i-1, j}}-F R A\left(t ; T_{i-1}, T_{i}\right)-R^{t}\right]
$$

Given that the cost of the CMS at time- $t$ is zero, we have,

$$
R^{t}=\frac{\sum_{i=1}^{n} P_{t}^{T_{i}} \delta\left[S_{t}^{t_{i-1, j}}+C A_{t}^{t_{i-1, j}}\right]}{\sum_{i=1}^{n} \delta P_{t}^{T_{i}}}-\frac{\sum_{i=1}^{n} \delta P_{t}^{T_{i}} F R A\left(t ; T_{i-1}, T_{i}\right)}{\sum_{i=1}^{n} \delta P_{t}^{T_{i}}}
$$

where the second part of the equation is the forward swap rate defined in Eq. (9).

The convexity adjustment $C A_{t}^{t_{i-1, j}}$ is determined by changing numeraire in the first term of Eq. (13) as,

$$
C A_{t}^{t_{i-1, j}}=\mathbb{E}^{Q_{d, j}^{t_{i, j}}}\left[S_{T_{i-1}^{t_{i-1, j}}}\left(\frac{G_{T_{i-1}}^{i}}{G_{t}^{i}}-1\right)\right]
$$

where for $i \in\{1, \ldots, n\}$ we have defined the function $G_{t}^{i}=\frac{P_{t}^{T_{i}}}{P_{t}^{t_{i, j}}}$. The convexity adjustment is approximated by approximating the $G_{t}^{i}$ term. 


\subsection{Flat term structure with parallel shifts}

Following Hagan (2005) and Brigo and Mercurio (2006), we initially derive an expression for the convexity adjustment when the term structure is flat and can only evolve with parallel shifts. We denote by $r_{t}$, the time- $t$ value (tenor $\tau$ ) spot rate. For $t \leq T_{i-1}$, the two numeraires are given as,

$$
P_{t}^{T_{i}}=\frac{1}{\left(1+\tau r_{t}\right)^{\Delta}} P_{t}^{T_{i-1}}
$$

and,

$$
P_{t}^{t_{i, j}}=\sum_{j=1}^{c} \tau \frac{1}{\left(1+\tau r_{t}\right)^{j}} P_{t}^{T_{i-1}}=P_{t}^{T_{i-1}} \frac{1}{r_{t}}\left[1-\frac{1}{\left(1+\tau r_{t}\right)^{c}}\right]
$$

Using Eqs. (18) and (19), function $G_{t}^{i}$ is given as,

$$
G_{t}^{i}=\frac{P_{t}^{T_{i}}}{P_{t}^{t_{i, j}}}=\frac{P_{t}^{T_{i-1}}(1+\tau r)^{-\Delta}}{P_{t}^{T_{i-1}} \frac{1}{r_{t}}\left[1-\frac{1}{\left(1+\tau r_{t}\right)^{c}}\right]}=\frac{r_{t}}{\left(1+\tau r_{t}\right)^{\Delta}} \frac{1}{1-\frac{1}{\left(1+\tau r_{t}\right)^{c}}}:=G\left(r_{t}\right)
$$

An important assumption we make when we work under multiple curves, is the fact that the swap rate is not a risk-free rate anymore. More specifically, following Liu et al. (2006) and Filipović and Trolle (2013) among others, we assume it to be equal to the risk-free rate $r_{t}$ (assumed to be the OIS rate) plus a spread $X_{t}$, written as,

$$
S_{t}^{t_{i, j}}=R_{t}=r_{t}+X_{t}
$$

where, $X_{t}$ is the spread incorporating the credit and liquidity risk premia of the counterparty and $R_{t}$ is the risky forward swap rate defined in Eq. (10). What is worth mentioning at this point, is the fact that under the assumption of a flat term structure, the forward rate $F R A\left(t ; t_{i, j}, t_{i, j+1}\right)$, does not depend on $j$ (i.e. assumed to be constant). We approximate $\mathrm{G}$ using a first-order Taylor expansion as,

$$
\frac{G\left(r_{T_{i-1}}\right)}{G\left(r_{t}\right)}-1 \approx \frac{G^{\prime}\left(r_{t}\right)}{G\left(r_{t}\right)}\left(r_{T_{i-1}}-r_{t}\right)=\frac{G^{\prime}\left(S_{t}^{t_{i-1, j}}-X_{t}\right)}{G\left(S_{t}^{t_{i-1, j}}-X_{t}\right)}\left[S_{T_{i-1}}^{t_{i-1, j}}-X_{T_{i-1}}-S_{t}^{t_{i-1, j}}+X_{t}\right]
$$

Using Eqs. (17) and (22), the convexity adjustment can be approximated as,

$$
\begin{aligned}
C A_{t}^{t_{i-1, j}}= & \frac{G^{\prime}\left(S_{t}^{t_{i-1, j}}-X_{t}\right)}{G\left(S_{t}^{t_{i-1, j}}-X_{t}\right)} \mathbb{E}^{t_{i, j}}\left[\left(S_{T_{i-1}}^{t_{i-1, j}}\right)^{2}-S_{T_{i-1}}^{t_{i-1, j}}\left(S_{t}^{t_{i-1, j}}+X_{T_{i-1}}-X_{t}\right)\right] \\
= & \left(S_{t}^{t_{i-1, j}}\right)^{2} \frac{G^{\prime}\left(S_{t}^{t_{i-1, j}}-X_{t}\right)}{G\left(S_{t}^{t_{i-1, j}}-X_{t}\right)} \times\left[\left(\mathbb{E}^{t_{d}^{t_{i, j}}}\left[\left(\frac{S_{T_{i-1}}^{t_{i-1, j}}}{S_{t}^{t_{i-1, j}}}\right)^{2}\right]-1\right)\right] \\
& -\left(\mathbb { E } _ { d } ^ { Q _ { i , j } } \left[\frac{\left.\left.\left.S_{T_{i-1}^{t_{i-1, j}} X_{T_{i-1}}}^{\left(S_{t}^{t_{i-1, j}}\right)^{2}}\right]-\frac{X_{t}}{S_{t}^{t_{i-1, j}}}\right)\right]}{}\right.\right.
\end{aligned}
$$

In the above expression, there are two expectations we need to calculate, $\mathbb{E} Q_{d}^{t_{i, j}}\left[\left(S_{T_{i-1}}^{t_{i-1, j}}\right)^{2}\right]$ and $\mathbb{E}_{d}^{t_{i, j}}\left[S_{T_{i-1}}^{t_{i-1, j}} X_{T_{i-1}}\right]$. We assume that under $Q_{d}^{t_{i, j}}$ the two processes (i.e. swap rate and spread), which are log-normal with constant volatility, are martingales and are of the form, 
$d S_{t}^{t_{i-1, j}}=\sigma_{t, S} S_{t}^{t_{i-1, j}} d W_{t, S}$, for the swap rate and $d X_{t}=\sigma_{t, X} X_{t} d W_{t, X}$, for the spread, where $W_{t, S}$ and $W_{t, X}$ are two correlated wiener processes with correlation $\rho_{s, x}$ and $\sigma_{t, S}$ and $\sigma_{t, X}$ are deterministic volatilities. Applying Ito's Lemma, the two expectations are given as (see “Appendix 1"),

$$
\begin{aligned}
\mathbb{E}^{Q_{i, j}^{t_{i, j}}}\left[\left(S_{T_{i-1}}^{t_{i-1, j}}\right)^{2}\right] & =\left(S_{t}^{t_{i-1, j}}\right)^{2} \exp \left(\left(\sigma_{t, S}\right)^{2}\left(T_{i-1}-t\right)\right) \\
\mathbb{E} Q_{d}^{t_{i, j}}\left[S_{T_{i-1}}^{t_{i-1, j}} X_{T_{i-1}}\right] & =S_{t}^{t_{i-1, j}} X_{t} \exp \left(\rho_{s, x} \sigma_{t, S} \sigma_{t, X}\left(T_{i-1}-t\right)\right)
\end{aligned}
$$

Using the two expectations, the convexity adjustment is given as,

$$
C A_{t}^{t_{i-1, j}}=K\left(r_{t}\right)\left[\left(e^{\left(\sigma_{t, S}\right)^{2}\left(T_{i-1}-t\right)}-1\right)-\frac{X_{t}}{S_{t}^{t_{i-1, j}}}\left(e^{\rho_{s, x} \sigma_{t, S} \sigma_{t, X}\left(T_{i-1}-t\right)}-1\right)\right]
$$

with,

$$
K\left(r_{t}\right)=\frac{\left(S_{t}^{t_{i-1, j}}\right)^{2}}{r_{t}} \frac{1}{1+\tau r_{t}}\left(1+(\tau-\delta) r_{t}-\frac{c \tau r_{t}}{\left(1+\tau r_{t}\right)^{c}-1}\right)
$$

Assuming that there is no spread in the market (i.e. if $X_{t}=0$ ), we end up with the well-known Black-like adjustment formula proposed by Hagan (2005).

\subsection{A term structure with tilts}

In this section, we depart from the restrictive and unrealistic assumption of a flat term structure and we extend our analysis by allowing for a tilt. Since we no longer assume a flat term structure, the spot rate $r_{t}^{T}$ is now given by some deterministic function $f$ as follows,

$$
r_{t}^{T}=f\left(r_{t}, t, T \mid a\right)
$$

where $r_{t}$ is the short rate and $a=\left(a_{1}, \ldots, a_{k}\right)$ is some vector of parameters. The $\mathrm{G}$ function we need to approximate is now given by,

$$
G_{t}^{i}=\frac{P_{t}^{T_{i}}}{P_{t}^{t_{i, j}}}=\frac{\left(1+\tau r_{t}^{T_{i}}\right)^{-\frac{\left(T_{i}-t\right)}{\tau}}}{\sum_{j=1}^{c} \tau\left(1+\tau r_{t}^{t_{i, j}}\right)^{-\frac{\left(t_{i, j}-t\right)}{\tau}}}=\frac{\left(1+\tau f_{t}^{T_{i}}\left(r_{t}\right)\right)^{-\frac{\left(T_{i}-t\right)}{\tau}}}{\sum_{j=1}^{c} \tau\left(1+\tau f_{t}^{t_{i, j}}\left(r_{t}\right)\right)^{-\frac{\left(t_{i, j}-t\right)}{\tau}}}
$$

where, for simplicity, we have written $f_{t}^{T_{i}}\left(r_{t}\right)$ for $r_{t}^{T_{i}}=f\left(r_{t}, t, T_{i}\right)$ and $f_{t}^{t_{i, j}}\left(r_{t}\right)$ for $r_{t}^{t_{i, j}}=$ $f\left(r_{t}, t, t_{i, j}\right)$. As in the flat term structure case, we approximate $\mathrm{G}$ using a first-order Taylor expansion at $\left(r_{t}, t\right)$ as,

$$
\frac{G\left(r_{T_{i-1}}, T_{i-1}\right)}{G\left(r_{t}, t\right)}-1 \approx \frac{G_{r}\left(r_{t}, t\right)}{G\left(r_{t}, t\right)}\left(r_{T_{i-1}}-r_{t}\right)+\frac{G_{t}\left(r_{t}, t\right)}{G\left(r_{t}, t\right)}\left(T_{i-1}-t\right)
$$

where $G_{r}$ and $G_{t}$ denote the partial derivatives of $G$ with respect to $r$ and $t$. In the previous case we assumed that $S_{t}^{t_{i, j}}=R_{t}$. In the current case, where we allow for a tilt in the term structure, we assume the following approximations, $S_{t}^{t_{i, j}} \approx R_{t}$ and $S_{T_{i-1}}^{t_{i, j}} \approx R_{T_{i-1}}$. 
Using Eqs. (17) and (30), the convexity adjustment can be approximated as,

$$
\begin{aligned}
C A_{t}^{t_{i-1, j}}= & \left(S_{t}^{t_{i-1, j}}\right)^{2} \frac{G_{r}\left(r_{t}, t\right)}{G\left(r_{t}, t\right)}\left[\left(\mathbb{E}^{Q_{d}^{t_{i, j}}}\left[\left(\frac{S_{T_{i-1}}^{t_{i-1, j}}}{S_{t}^{t_{i-1, j}}}\right)^{2}\right]-1\right)\right] \\
& -\left(S_{t}^{t_{i-1, j}}\right)^{2} \frac{G_{r}\left(r_{t}, t\right)}{G\left(r_{t}, t\right)}\left[\left(\mathbb{E}^{Q_{d}^{t_{i, j}}}\left[\frac{S_{T_{i-1}}^{t_{i-1, j}} X_{T_{i-1}}}{\left(S_{t}^{t_{i-1, j}}\right)^{2}}\right]-\frac{X_{t}}{S_{t}^{t_{i-1, j}}}\right)\right] \\
& +\frac{G_{t}\left(r_{t}, t\right)}{G\left(r_{t}, t\right)} S_{t}^{t_{i-1, j}}\left(T_{i-1}-t\right)
\end{aligned}
$$

As before, we assume that under $Q_{d}^{t_{i, j}}$ the two (log-normal with constant volatility) processes (i.e. swap rate and spread) are martingales and the expectations are given by Eqs. (24) and (25). So, the convexity adjustment is now given by,

$$
\begin{aligned}
C A_{t}^{t_{i-1, j}}= & \left(S_{t}^{t_{i-1, j}}\right)^{2} \frac{G_{r}\left(r_{t}, t\right)}{G\left(r_{t}, t\right)}\left[\left(e^{\left(\sigma_{t, S}\right)^{2}\left(T_{i-1}-t\right)}-1\right)-\frac{X_{t}}{S_{t}^{t_{i-1, j}}}\left(e^{\rho_{s, x} \sigma_{t, S} \sigma_{t, X}\left(T_{i-1}-t\right)}-1\right)\right] \\
& +\frac{G_{t}\left(r_{t}, t\right)}{G\left(r_{t}, t\right)} S_{t}^{t_{i-1, j}}\left(T_{i-1}-t\right)
\end{aligned}
$$

We also need to calculate the two terms $\frac{G_{r}(r, t)}{G(r, t)}$ and $\frac{G_{t}(r, t)}{G(r, t)}$ that incorporate the partial derivatives. Analytical expressions are given in "Appendix 2".

Finally, for function $f$ we use the following parametric functional form, based on the well-known Nelson and Siegel model.

$$
f(r, t, T \mid a, b, k)=r+(a+b(T-t)) e^{-k(T-t)}-a
$$

with,

$$
\left.\begin{array}{l}
\frac{\partial f_{t}^{T_{i}}}{\partial r}(r)=\frac{\partial f_{t}^{t_{i, j}}}{\partial r}(r)=1 \\
\frac{\partial f_{t}^{T_{i}}}{\partial t}(r)=\left(k\left(a+b\left(T_{i}-t\right)\right)-b\right) e^{-k\left(T_{i}-t\right)} \\
\frac{\partial f_{t}^{t_{i, j}}}{\partial t}(r)=\left(k\left(a+b\left(t_{i, j}-t\right)\right)-b\right) e^{-k\left(t_{i, j}-t\right)}
\end{array}\right\}
$$

\section{Smile-consistent convexity adjustment}

In order to test the proposed CMS convexity adjustments, we compare them with the smileconsistent convexity adjustment, which is widely used in the market. In the presence of a market smile, when the term structure is not flat, but may tilt, the adjustment is necessarily more involved, if we aim to incorporate consistently the information coming from the quoted implied volatilities. The procedure to derive a smile consistent convexity adjustment is described in Mercurio and Pallavicini (2006) and Pallavicini and Tarenghi (2010), and is the one we will use here.

For the consistent derivation of CMS convexity adjustment, volatility modelling is required. We use the SABR model (a popular market choice for swaption smile analysis) for the swap rate in order to infer from it the volatility smile surface. The SABR model assumes that $S_{t}^{t_{i-1, j}}$ evolves under the associated forward swap measure $Q_{d}^{t_{i, j}}$ according to, 


$$
\left.\begin{array}{l}
d S_{t}^{t_{i-1, j}}=V_{t}\left(S_{t}^{t_{i-1, j}}\right)^{\beta} d Z_{t}^{t_{i-1, j}} \\
d V_{t}=\epsilon V_{t} d W_{t}^{t_{i-1, j}} \\
V_{0}=\alpha
\end{array}\right\}
$$

where, $Z_{t}^{t_{i-1, j}}$ and $W_{t}^{t_{i-1, j}}$ are $Q_{d}^{t_{i, j}}$-standard Brownian motions with,

$$
d Z_{t}^{t_{i-1, j}} d W_{t}^{t_{i-1, j}}=\rho d t
$$

and where $\beta \in(0,1], \epsilon$ and $\alpha$ are positive constants and $\rho \in[-1,1]$. The CMS convexity adjustment is given in Mercurio and Pallavicini (2006) as,

$$
\begin{aligned}
& C A^{S A B R}\left(S_{t}^{t_{i-1, j}} ; \delta\right) \\
& =\theta\left(S_{0}^{t_{i-1, j}}\right)\left(\frac{2}{\left(S_{0}^{t_{i-1, j}}\right)^{2}} \int_{0}^{\infty} \operatorname{Black}\left(K, S_{0}^{t_{i-1, j}}, v^{i m p}\left(K, S_{0}^{t_{i-1, j}}\right)\right) d K-1\right)
\end{aligned}
$$

where,

$$
\begin{aligned}
& \operatorname{Black}(K, S, v):=S \Phi\left(\frac{\ln (S / K)+v^{2} / 2}{v}\right)-K \Phi\left(\frac{\ln (S / K)-v^{2} / 2}{v}\right) \\
& v^{i m p}\left(K, S_{0}^{t_{i-1, j}}\right):=\sigma^{i m p}\left(K, S_{0}^{t_{i-1, j}}\right) \sqrt{T_{i-1}}
\end{aligned}
$$

An approximation for the implied volatility of the swaption with maturity $T_{i-1}$ is derived in Hagan et al. (2002) as,

$$
\begin{aligned}
\sigma^{i m p}\left(K, S_{0}^{t_{i-1, j}}\right) \approx & \frac{\alpha}{\left(S_{0}^{t_{i-1, j}} K\right)^{\frac{1-\beta}{2}}\left[1+\frac{(1-\beta)^{2}}{24} \ln ^{2}\left(\frac{s_{0}^{t_{i-1, j}}}{K}\right)+\frac{(1-\beta)^{4}}{1920} \ln ^{4}\left(\frac{s_{0}^{t_{i-1, j}}}{K}\right)\right]} \frac{z}{x(z)} \\
& \cdot\left\{1+\left[\frac{(1-\beta)^{2} \alpha^{2}}{24\left(S_{0}^{t_{i-1, j}} K\right)^{1-\beta}}+\frac{\rho \beta \epsilon \alpha}{4\left(S_{0}^{t_{i-1, j}} K\right)^{\frac{1-\beta}{2}}}+\epsilon^{2} \frac{2-3 \rho^{2}}{24}\right] T_{i-1}\right\}
\end{aligned}
$$

where

$$
z:=\frac{\epsilon}{\alpha}\left(S_{0}^{t_{i-1, j}} K\right)^{\frac{1-\beta}{2}} \ln \left(\frac{S_{0}^{t_{i-1, j}}}{K}\right)
$$

and

$$
x(z):=\ln \left\{\frac{\sqrt{1+2 \rho z+z^{2}}+z-\rho}{1-\rho}\right\}
$$

The above formula provides us with an efficient approximation for the SABR implied volatility for each strike $K$. We consider a different SABR model for each swap rate contained in the CMS payoff and we perform a calibration of all the SABR parameters (four parameters $(\alpha, \beta, \rho, \epsilon)$ for each swap rate) to swaption volatility smile and CMS spread quoted in the market. See Mercurio and Pallavicini (2006) and Pallavicini and Tarenghi (2010) for a detailed description of the calibration procedure. 


\section{Market data}

We use three data sets for this study, one containing Euro money market instruments for the construction of the yield curves, a second one containing CMS swap spreads with a maturity of 5-years, where the associated underlying swaps have a 10 -year maturity (i.e. $X_{5,10}$ ), and a third one containing swaption volatilities for different strikes, as well as implied black at-the-money (ATM) swaption volatilities. All market data was collected from Bloomberg. Data sets are presented in detail below:

- For the discounting curve, we use Eonia Fixing and OIS rates from 3-months to 30-years.

- For the 3-month curve, we use Euribor 6-months fixing, FRA rates up to 15 months, and swaps from 2 to 30 years, paying an annual fix rate in exchange for the Euribor 3-month rate.

- For the 6-month curve, we use Euribor 6-months fixing, FRA rates up to 2 years, and swaps from 2 to 30 years, paying an annual fix rate in exchange for the Euribor 6-month rate.

The market quotes a value for the CMS spread which makes the CMS swap fair. However, it quotes the spread only for a few CMS swap maturities and tenors (usually 5, 10, 15, 20 and 30 years). In the Euro market, the CMS tenor is equal to 3 months, while the $c$-year IRS which is used as indexation in the CMS has Libor payments of 6-months or 1-year frequency. Thus, CMS spreads depend on three different curves in our framework; first, the funding curve used to discount the cash flows of the CMS swap, which we consider to be the risk-free curve (i.e. OIS curve); second, the 3-month forwarding curve for the Euribor rates paid in the second leg of the CMS; and third, the 6-month (or 1-year) forwarding curve for the Euribor rates paid by the indexation IRS.

\section{Empirical results}

In this section, we compare numerically the accuracy of the approximations for the CMS convexity adjustments against the Black and SABR models convexity adjustments presented in Sect. 4.

\subsection{An empirical illustration}

Our first numerical example is based on Euro data as of 3 February 2006. We test a CMS with maturity of 5 years (i.e. $n \delta=5$ ), where the associated underlying swaps have maturity of 10 years (i.e. $c \tau=10$ ). The closing price for the CMS spread is $X_{5,10}=64.9$ basis points (bps). The ATM swaption volatility is $\sigma_{5,10}^{A T M}=0.15$, and swaption volatilities for different strikes are given in Table 1. For the parameters of the term structure in case 2, we choose the values: $(a, b, k)=(0.01,0.002,0.1)$. Finally, when we apply the case with the spread, we assume that the spread is constant at $X_{t}=100 \mathrm{bps}$, while its volatility is $\sigma_{t, x}=0.1$, and the correlation is $\rho_{s, x}=0.9$. The calibration procedure is performed by minimising the square difference between CMS spreads (and swaption volatilities) and the market data. Our results are summarized in Tables 2, 3 and 4. We denote by case 1, the Black-like (flat term structure) convexity adjustment of Eq. (26) and by case 2, the (tilt term structure) convexity adjustment of Eq. (31). For the Black-like convexity adjustment, we set $X_{t}=0$, in Eq. (26), while for the SABR model, we use Eq. (36). 
Table 1 This table reports market quotes of swaption volatilities (in \%) for different strikes $K$

\begin{tabular}{llllllllll}
\hline Expiry & Tenor & -200 & -100 & -50 & -25 & 25 & 50 & 100 & 200 \\
\hline 5 years & 10years & $6.54 \%$ & $2.30 \%$ & $0.93 \%$ & $0.41 \%$ & $-0.30 \%$ & $-0.51 \%$ & $-0.68 \%$ & $-0.39 \%$ \\
\hline
\end{tabular}

Each strike indicates the difference from the ATM volatility

Table 2 This table presents the market price of the CMS spread against the calibrated price of the spread using the (case 1) Black-like convexity adjustment (parallel shift), the (case 2) second convexity adjustment (allows for tilt) and the SABR model

\begin{tabular}{lllll}
\hline & Market & Case 1 & Case 2 & SABR \\
\hline Price & 0.00649 & 0.006237 & 0.006402 & 0.006406 \\
Difference (in bps) & & 2.53 & 0.89 & 0.83 \\
\hline
\end{tabular}

The differences between the market price and the three different models are provided in basis points (bps). In this case the spread, $X_{t}$ of the swap rate is not taken into account

Table 3 This table presents the market price of the CMS spread against the calibrated price of the spread using the (case 1) Black-like convexity adjustment (parallel shift), the (case 2) second convexity adjustment (tilt) and the SABR model

\begin{tabular}{|c|c|c|c|c|}
\hline & Market & Case 1 & Case 2 & SABR \\
\hline Price & 0.00649 & 0.006352 & 0.0065001 & 0.006406 \\
\hline Difference (in bps) & & 1.38 & 0.1 & 0.83 \\
\hline
\end{tabular}

The differences between the market price and the three different models are provided in basis points (bps). We incorporate the swap spread, $X_{t}$, by assuming that the swap rate is equal to the risk-free rate plus the spread

Our numerical results suggest that in all cases market data are well reproduced. As Table 2 reports, the SABR model performs slightly better than our new convexity adjustment (case 2 ), with $0.89 \mathrm{bps}$ compared to $0.83 \mathrm{bps}$, when the spread is not taken into account, and much better compared to the Black-like formula (case 1), 0.83 bps against 2.53 bps. However, this is not the case when we take into account the swap spread. The absolute difference (in bps) between our new convexity adjustment model and the market is significantly smaller compared to the SABR case, i.e. 0.1 bps compared to 0.83 bps. Furthermore, as expected, Black's model calibration results, although better than the non-spread case (1.38 bps against $2.53 \mathrm{bps}$ ), still fail to fit the data compared to the other two cases. In addition, in Table 4, we report the convexity adjustments for all four cases. We observe that the convexity adjustment in the 'tilt' case is significantly larger than the 'flat' case, especially, when the swap spread is incorporated. Furthermore, in the non-flat case, convexity adjustment presents a curvy shape compared to the earlier Black-like case, where the shape behaves in a more static way.

\subsection{Numerical examples}

In order to further test the accuracy of the approximations for the CMS convexity adjustments against the Black and SABR models convexity adjustments, we calibrate the models to different dates spanning the period from 2007 to 2012. This period covers the most interesting phases of the unfolding of the global financial crisis and, as such, we can derive safer conclusions of how the proposed convexity adjustments perform under different market conditions (i.e. periods of stability and market turmoil). In Table 5, we present market data for CMS 
Table 4 This table presents the convexity adjustment for all four different cases, i.e. the Black-like 'flat' term structure with and without spread, and the 'tilt' term structure with and without spread

\begin{tabular}{|c|c|c|c|c|}
\hline $\mathrm{i}$ & CA (case 1) & CA (case 2) & CA (case 1 -with spread) & CA (case 2 -with spread) \\
\hline 1 & 0.000036 & 0.000032 & 0.000037 & 0.000033 \\
\hline 2 & 0.000074 & 0.000083 & 0.000076 & 0.000092 \\
\hline 3 & 0.000114 & 0.000198 & 0.000116 & 0.000218 \\
\hline 4 & 0.000154 & 0.000313 & 0.000158 & 0.000343 \\
\hline 5 & 0.000196 & 0.000428 & 0.000201 & 0.000469 \\
\hline 6 & 0.000238 & 0.000542 & 0.000245 & 0.000593 \\
\hline 7 & 0.000282 & 0.000656 & 0.000289 & 0.000718 \\
\hline 8 & 0.000326 & 0.000769 & 0.000335 & 0.000842 \\
\hline 9 & 0.000373 & 0.000883 & 0.000383 & 0.000967 \\
\hline 10 & 0.000420 & 0.000995 & 0.000431 & 0.001090 \\
\hline 11 & 0.000468 & 0.001107 & 0.000480 & 0.001213 \\
\hline 12 & 0.000514 & 0.001216 & 0.000528 & 0.001334 \\
\hline 13 & 0.000564 & 0.001326 & 0.000579 & 0.001455 \\
\hline 14 & 0.000615 & 0.001436 & 0.000631 & 0.001576 \\
\hline 15 & 0.000667 & 0.001544 & 0.000685 & 0.001697 \\
\hline 16 & 0.000721 & 0.001652 & 0.000739 & 0.001817 \\
\hline 17 & 0.000775 & 0.001759 & 0.000795 & 0.001936 \\
\hline 18 & 0.000831 & 0.001865 & 0.000852 & 0.002054 \\
\hline 19 & 0.000888 & 0.001970 & 0.000911 & 0.002172 \\
\hline 20 & 0.000946 & 0.002074 & 0.000970 & 0.002289 \\
\hline
\end{tabular}

The whole structure from year 1 to year 20 is given

Table 5 This table reports market CMS swap spreads (in bps) and market ATM swaption volatilities data for specific dates

CMS swap spreads have cms maturity of 5 years and associated underlying swaps with maturity of 10 years

\begin{tabular}{lll}
\hline Date & Market CMS & Market volatility (\%) \\
\hline $10 / 08 / 2007$ & 41.6 & 12.30 \\
$31 / 10 / 2008$ & 104.5 & 12.98 \\
$28 / 05 / 2010$ & 178.1 & 19.30 \\
$03 / 06 / 2011$ & 136.4 & 19.80 \\
$09 / 03 / 2012$ & 158.65 & 25.15 \\
\hline
\end{tabular}

swap spreads, where the maturity of the CMS is 5 years, and the associated underlying swaps have a 10-year maturity. Furthermore, the market at-the-money swaption volatilities, for all different dates, are reported, while Table 6 reports market volatility smiles across different strikes and for different dates. We can observe that market data clearly show levels of turmoil in the market during the period of the financial crisis.

In Table 7, we report all calibrated parameters. Our results for different dates and market data are summarized in Table 8 , where all prices are given in basis points. Our numerical results suggest that in each case and in each period, the convexity adjustment in the case of the 'tilt' term structure (with swap spread incorporated), gives better results in terms of fitting market CMS spreads. Our new convexity adjustment (tilt), gives sufficiently accurate and robust results across all market scenarios (stability and turmoil) and spread levels. 
Table 6 This table reports market swaption volatility smiles for different dates

\begin{tabular}{lllllllll}
\hline Date & $-200(\%)$ & $-100(\%)$ & $-50(\%)$ & $-25(\%)$ & $25(\%)$ & $50(\%)$ & $100(\%)$ & $200(\%)$ \\
\hline $10 / 08 / 2007$ & 2.75 & 1.01 & 0.44 & 0.20 & -0.18 & -0.29 & -0.47 & -0.70 \\
$31 / 10 / 2008$ & 3.81 & 1.08 & 0.40 & 0.20 & -0.20 & -0.40 & -0.72 & -1.22 \\
$28 / 05 / 2010$ & 8.53 & 3.12 & 1.29 & 0.64 & -0.45 & -0.86 & -1.38 & -2.09 \\
$03 / 06 / 2011$ & 6.96 & 2.51 & 1.09 & 0.46 & -0.42 & -0.76 & -1.22 & -1.78 \\
$09 / 03 / 2012$ & 9.61 & 3.02 & 1.23 & 0.56 & -0.44 & -0.77 & -1.30 & -1.81 \\
\hline
\end{tabular}

Strikes are expressed as absolute differences in basis points w.r.t. the ATM values

Table 7 This table presents the model parameters, obtained from the calibrated procedure, of the functions described in Sects. 3 and 4 for the different dates in our sample

\begin{tabular}{lccccc}
\hline Date & $10 / 08 / 2007$ & $31 / 10 / 2008$ & $28 / 05 / 2010$ & $03 / 06 / 2011$ & $09 / 03 / 2012$ \\
\hline SABR parameters & & & & & \\
$\quad$ alpha & 0.0585 & 0.0579 & 0.0885 & 0.0912 & 0.1139 \\
beta & 0.7539 & 0.7161 & 0.768 & 0.7643 & 0.7794 \\
rho & -0.2341 & -0.277 & -0.3957 & -0.3433 & -0.2364 \\
epsilon & 0.1926 & 0.2177 & 0.2848 & 0.2867 & 0.231 \\
f (Nelson-Siegel) & & & & -0.01 & -0.002 \\
a & 0.001 & 0.005 & -0.03 & 0.0043 & 0.0038 \\
b & 0.002 & 0.0012 & 0.0047 & 0.05 & 0.004 \\
k & 0.003 & 0.015 & 0.001 & & \\
\hline
\end{tabular}

Table 8 This table compares market CMS spread prices against each different case

\begin{tabular}{llllcc}
\hline Date & $10 / 08 / 2007$ & $31 / 10 / 2008$ & $28 / 05 / 2010$ & $03 / 06 / 2011$ & $09 / 03 / 2012$ \\
\hline Market & 41.6 & 104.5 & 178.1 & 136.4 & 158.65 \\
Case 1 (Flat) & 37.92 & 112.88 & 165.78 & 125.73 & 151.16 \\
Case 2 (Tilt) & 40.94 & 107.73 & 173.15 & 131.29 & 154.99 \\
SABR & 39.95 & 111.77 & 168.81 & 128.21 & 152.5 \\
(Mkt-SABR) & 1.65 & 7.27 & 9.29 & 8.19 & 6.15 \\
(Mkt-Tilt) & 0.66 & 3.23 & 4.95 & 5.11 & 3.66 \\
\hline
\end{tabular}

Absolute differences (in bps) between market CMS swap spreads and the models are given

Even in the period of 2008-2011, where market was experiencing an unprecedented turbulence, our convexity adjustment performs well, since the difference between the market data and the SABR model is sufficiently higher than the case of the 'tilt' term structure, with a difference of around 3-5 basis points (for the 'tilt') compared to 7-9 basis points (for the SABR). Furthermore, in every case the results are in between the limits of the bid-ask spread of around 10 basis points, indicating that the market data are well recovered across all periods.

Finally, convexity adjustments for all different cases, the (Black-like) flat term structure in red colour, the 'tilt' term structure in green colour and the SABR model in blue colour, are presented in Figs. 1, 2, 3, 4, 5 and 6. All cases take into account the spread 

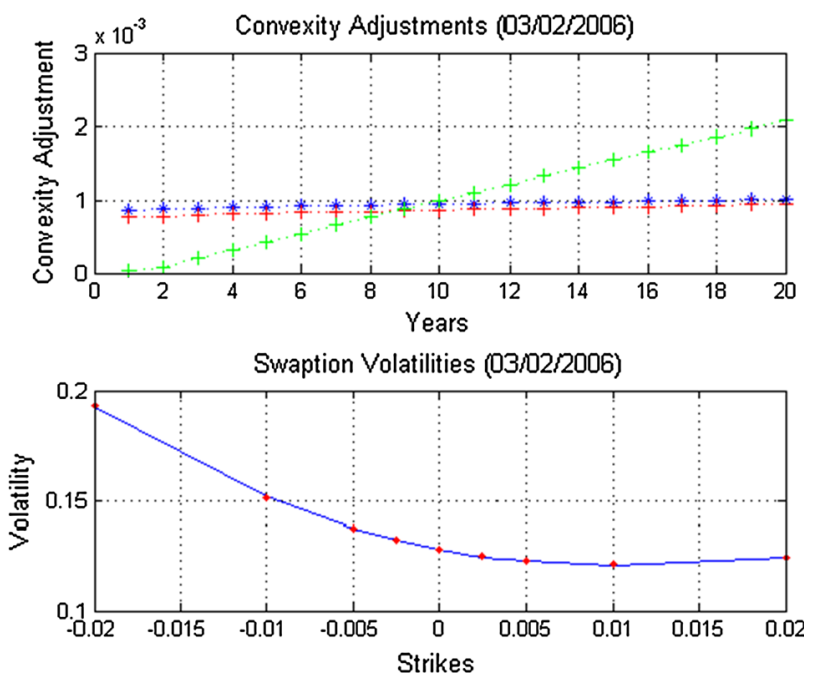

Fig. 1 This figure shows the convexity adjustments for the 'flat' term structure ( red), the 'tilt' term structure (green) and the SABR model (blue). In all cases the swap spread is taken into account. In the lower panel, the calibrated volatility smile is displayed. Results are from a specific date, 03/02/2006. (Colour figure online)
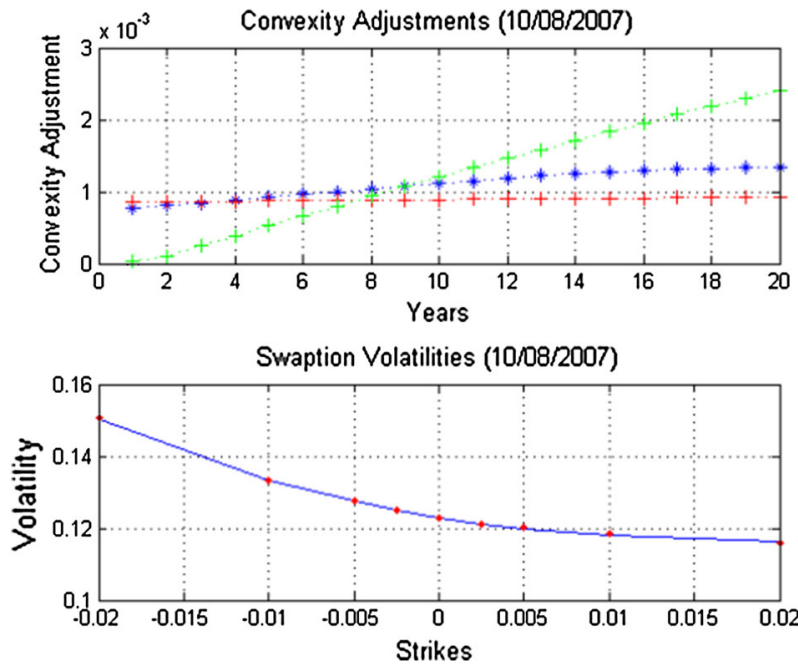

Fig. 2 This figure shows the convexity adjustments for the 'flat' term structure ( $r e d)$, the 'tilt' term structure (green) and the SABR model (blue). In all cases the swap spread is taken into account. In the lower panel, the calibrated volatility smile is displayed. Results are from a specific date, 10/08/2007. (Colour figure online)

on the swap rate. Furthermore, the outcome of the calibration procedure under the SABR model (i.e. the whole volatility smile against different strikes) is presented in the lower panel of the figures, where we observe that the SABR model is perfectly calibrated across different dates. The only exception is October of 2008, i.e. the peak of the financial crisis, where markets were under severe pressures, that the SABR model struggles to fit the volatility smile. Regarding the convexity adjustments, in all cases and across different periods, we depict similar characteristics. We observe that convexity adjustment with 'tilt' term 

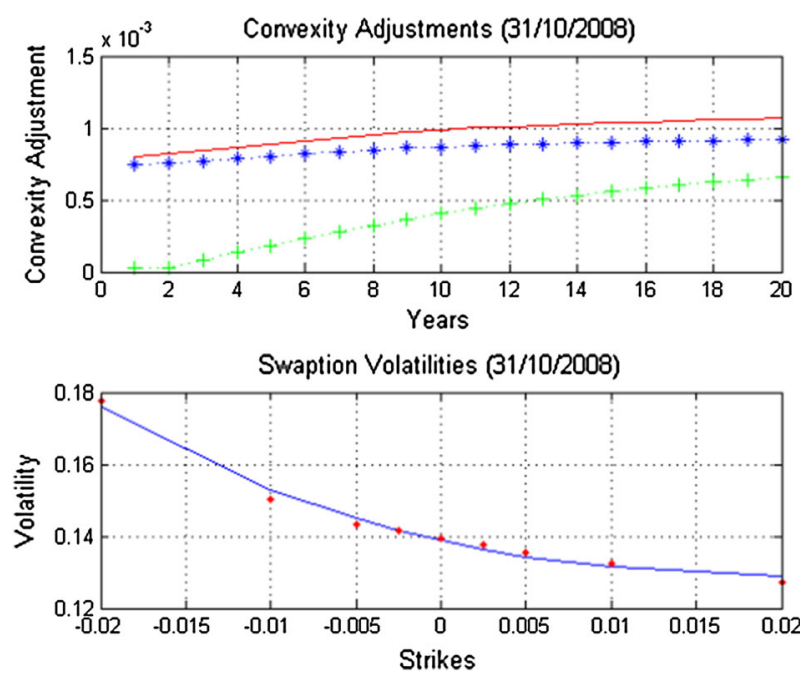

Fig. 3 This figure shows the convexity adjustments for the 'flat' term structure (red), the 'tilt' term structure (green) and the SABR model (blue). In all cases the swap spread is taken into account. In the lower panel, the calibrated volatility smile is displayed. Results are from a specific date, 31/10/2008. (Colour figure online)
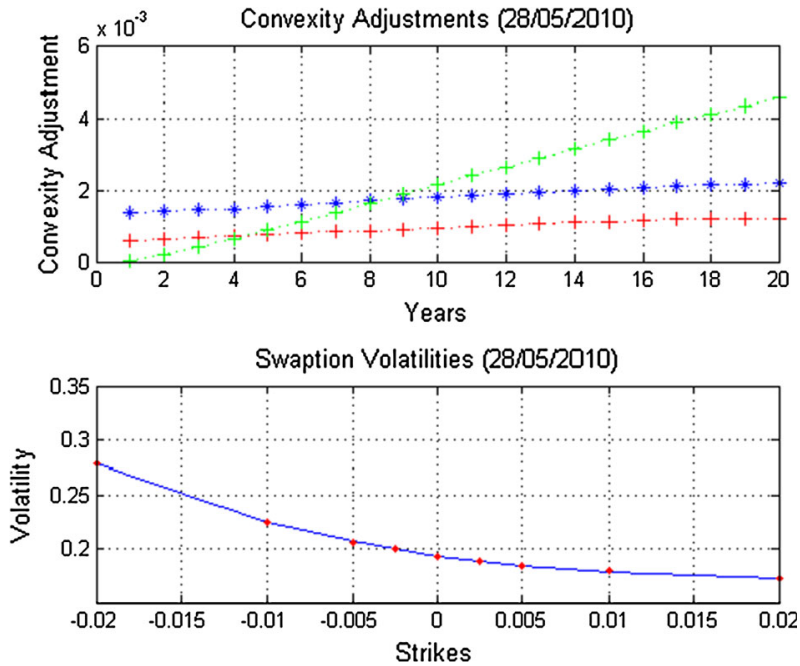

Fig. 4 This figure shows the convexity adjustments for the 'flat' term structure ( red), the 'tilt' term structure (green) and the SABR model (blue). In all cases the swap spread is taken into account. In the lower panel, the calibrated volatility smile is displayed. Results are from a specific date, 28/05/2010. (Colour figure online)

structure is significantly larger than in the other two cases. Furthermore, the shape of the non-flat case presents a slope compared to the Black-like case where the convexity adjustments are flat and static. This helps the model perform well, especially in periods of market turmoil. 


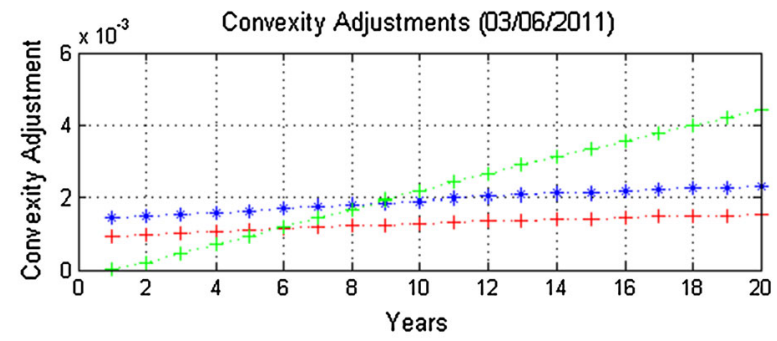

Swaption Volatilities (03/06/2011)

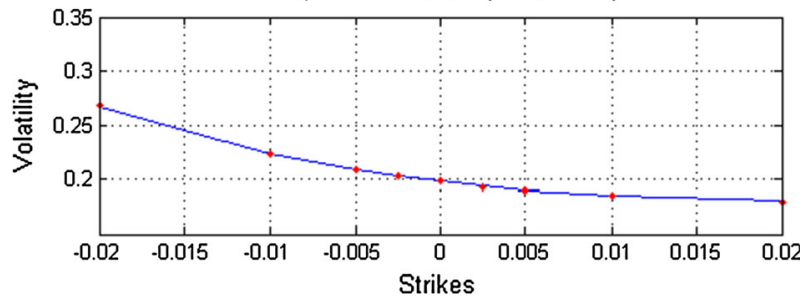

Fig. 5 This figure shows the convexity adjustments for the 'flat' term structure ( red), the 'tilt' term structure (green) and the SABR model (blue). In all cases the swap spread is taken into account. In the lower panel, the calibrated volatility smile is displayed. Results are from a specific date, 03/06/2011. (Colour figure online)
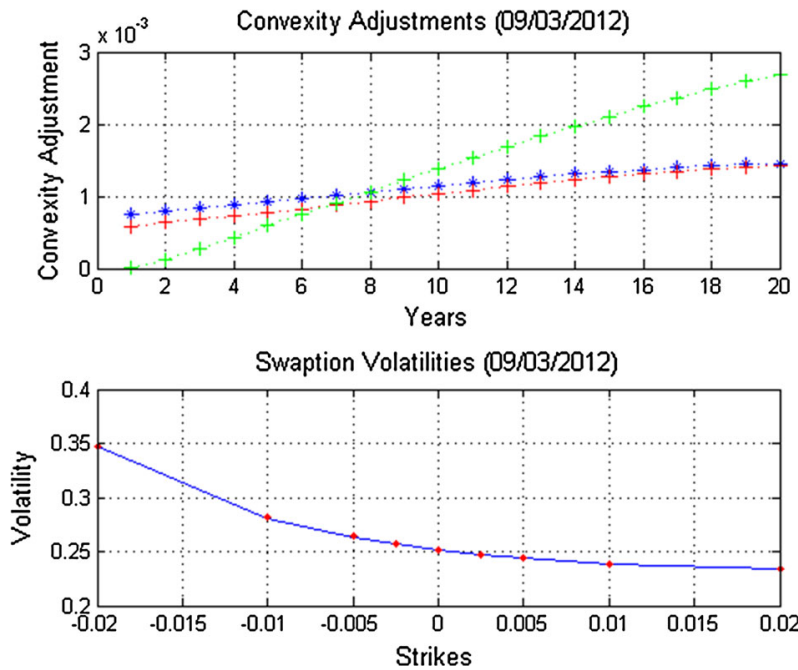

Fig. 6 This figure shows the convexity adjustments for the 'flat' term structure (red), the 'tilt' term structure (green) and the SABR model (blue). In all cases the swap spread is taken into account. In the lower panel, the calibrated volatility smile is displayed. Results are from a specific date, 09/03/2012. (Colour figure online)

\section{Conclusion}

In this paper we have developed a new CMS convexity adjustment in a double-curve framework, that separates the discounting and forwarding term structures. The motivation of our study comes from the unprecedented increase in the Libor-OIS spread that was experienced during the financial crisis, which has questioned the legitimacy of considering both (Libor and OIS) quotes as risk-free, and has raised valid issues in the construction of zero-coupon 
curves, which clearly, can no longer be based on traditional bootstrapping procedures. In that vein, our work fills the gap of the shortcomings of single yield curve model adjustments, widely used in the literature, when one deals with the issue of convexity in money market instruments.

In the double-curving environment that we describe, we have derived the convexity factor requirement in the conventional case that the term structure of interest rates is flat, and its dynamic evolution allows only for parallel shifts, and we have expanded our setting to incorporate the more realistic and challenging case of term structure tilts. The new term appears to be approximately linear in this parameter. In all computations, our results conclude that the convexity adjustment of the 'tilt' term structure case is significantly larger than the convexity adjustments implied by the Black and SABR models.

As an empirical illustration, we have calibrated both convexity adjustments to real market data, by using swaption volatilities, and calculated the differences between market quotes and our model implied CMS spreads. We further compared our results with the widely used by market practitioners smile-consistent CMS adjustment, using the SABR model. We considered a different SABR model for each swap rate contained in the CMS payoff, and we performed a calibration of all the SABR parameters to swaption volatility smile and CMS spreads quoted by the market. In all cases the swaption volatility smiles are very well recovered by the calibrated SABR models. Furthermore, our results demonstrate that the proposed convexity adjustments offer a market consistent and robust valuation of CMS spreads, and suggest that CMS-type of products should be priced under a multi-curve framework.

Open Access This article is distributed under the terms of the Creative Commons Attribution 4.0 International License (http://creativecommons.org/licenses/by/4.0/), which permits unrestricted use, distribution, and reproduction in any medium, provided you give appropriate credit to the original author(s) and the source, provide a link to the Creative Commons license, and indicate if changes were made.

\section{Appendix 1: Expectation of swap rate and spread}

To calculate $\mathbb{E}^{Q_{d}^{t_{i, j}}}\left[S_{T_{i-1}}^{t_{i-1, j}} X_{T_{i-1}}\right]$, we let $Y_{t}=\ln \left(S_{t}^{t_{i-1, j}} X_{t}\right)$, and we apply Ito's Lemma.

$$
\begin{aligned}
d Y_{t}= & \frac{\partial Y_{t}}{\partial S_{t}^{t_{i-1, j}}} d S_{t}^{t_{i-1, j}}+\frac{\partial Y_{t}}{\partial X_{t}} d X_{t}+\frac{1}{2} \frac{\partial^{2} Y_{t}}{\partial S_{t}^{t_{i-1, j}}}\left(d S_{t}^{t_{i-1, j}}\right)^{2} \\
& +\frac{1}{2} \frac{\partial^{2} Y_{t}}{\partial X_{t}^{2}}\left(d X_{t}\right)^{2}+\frac{\partial^{2} Y_{t}}{\partial S_{t}^{t_{i-1, j}} \partial X_{t}} d S_{t}^{t_{i-1, j}} d X_{t} \\
= & \sigma_{t, S} d W_{t, S}+\sigma_{t, X} d W_{t, X}-\frac{1}{2}\left[\left(\sigma_{t, S}\right)^{2}+\left(\sigma_{t, X}\right)^{2}\right] d t
\end{aligned}
$$

So,

$$
d \ln \left(S_{t}^{t_{i-1, j}} X_{t}\right)=\sigma_{t, S} d W_{t, S}+\sigma_{t, X} d W_{t, X}-\frac{1}{2}\left[\left(\sigma_{t, S}\right)^{2}+\sigma_{t, X}^{2}\right] d t
$$

which means that,

$$
S_{T_{i-1}}^{t_{i-1, j}} X_{T_{i-1}}=S_{t}^{t_{i-1, j}} X_{t} e^{-\frac{1}{2}\left[\left(\sigma_{t, S}\right)^{2}+\sigma_{t, X}^{2}\right]\left(T_{i-1}-t\right)} e^{\left[\sigma_{t, S} W_{T_{i-1, S}}+\sigma_{t, X} W_{T_{i-1, X}}\right]}
$$


So,

$$
\begin{aligned}
\mathbb{E}_{d}^{Q_{i, j}^{t_{i, j}}\left[S_{T_{i-1}}^{t_{i-1, j}} X_{T_{i-1}}\right]=} & \mathbb{E}^{Q_{d}^{t_{i, j}}}\left[S_{t}^{t_{i-1, j}} X_{t} e^{-\frac{1}{2}\left[\left(\sigma_{t, S}\right)^{2}+\sigma_{t, X}^{2}\right]\left(T_{i-1}-t\right)}\right] \\
& \times \mathbb{E}^{Q_{d}^{t_{i, j}}}\left[e^{\sigma_{t, S} W_{\left(T_{i-1}-t\right), S}+\sigma_{t, X} W_{\left(T_{i-1}-t\right), X}}\right]
\end{aligned}
$$

In general, if: $X \sim N\left(0, \sigma_{X}^{2}\right), Y \sim N\left(0, \sigma_{Y}^{2}\right)$ and $E\left[e^{X+Y}\right]$, then we have, $E\left[e^{\frac{1}{2} \operatorname{var}(X+Y)}\right]$ with $\operatorname{var}(X+Y)=\operatorname{var}(X)+\operatorname{var}(Y)+2 \rho \sqrt{\operatorname{var}(X) \operatorname{var}(Y)}$

So, in our case,

$$
\begin{aligned}
X & =\sigma_{t, S} W_{\left(T_{i-1}-t\right), S} \sim N\left(0,\left(\sigma_{t, S}\right)^{2}\left(T_{i-1}-t\right)\right) \\
Y & =\sigma_{t, X} W_{\left(T_{i-1}-t\right), X} \sim N\left(0, \sigma_{t, X}^{2}\left(T_{i-1}-t\right)\right) \\
\operatorname{var}(X+Y) & =\left(\sigma_{t, S}\right)^{2}\left(T_{i-1}-t\right)+\sigma_{t, X}^{2}\left(T_{i-1}-t\right)+2 \rho_{s, x} \sqrt{\left(\sigma_{t, S}\right)^{2} t \sigma_{t, X}^{2} t} \\
& =\left(\sigma_{t, S}\right)^{2}\left(T_{i-1}-t\right)+\sigma_{t, X}^{2}\left(T_{i-1}-t\right)+2 \rho_{s, x} \sigma_{t, S} \sigma_{t, X}\left(T_{i-1}-t\right)
\end{aligned}
$$

So,

$$
\mathbb{E} Q_{d}^{t_{i, j}}\left[e^{\sigma_{t, S} W_{\left(T_{i-1}-t\right), S}+\sigma_{t, X} W_{\left(T_{i-1}-t\right), X}}\right]=\mathbb{E}^{Q_{d}^{t_{i, j}}}\left[e^{\frac{1}{2}\left(\left(\sigma_{t, S}\right)^{2}+\sigma_{t, X}^{2}+2 \rho_{s, x} \sigma_{t, S} \sigma_{t, X}\right)\left(T_{i-1}-t\right)}\right]
$$

So, finally,

$$
\mathbb{E}^{Q_{d}^{t_{i, j}}}\left[S_{T_{i-1}}^{t_{i-1, j}} X_{T_{i-1}}\right]=S_{t}^{t_{i-1, j}} X_{t} e^{\rho_{s, x} \sigma_{t, S} \sigma_{t, X}\left(T_{i-1}-t\right)}
$$

\section{Appendix 2: Partial derivatives}

Calculation of partial derivatives: $\frac{G_{r}(r, t)}{G(r, t)}$ and $\frac{G_{t}(r, t)}{G(r, t)}$. We start with our function,

$$
G(r, t)=\frac{\left(1+\tau f_{t}^{T_{i}}(r)\right)^{-\frac{\left(T_{i}-t\right)}{\tau}}}{\sum_{j=1}^{c} \tau\left(1+\tau f_{t}^{t_{i, j}}(r)\right)^{-\frac{\left(t_{i, j}-t\right)}{\tau}}}
$$

and let,

$$
\begin{aligned}
& u=\left(1+\tau f_{t}^{T_{i}}(r)\right)^{-\frac{\left(T_{i}-t\right)}{\tau}} \\
& v=\sum_{j=1}^{c} \tau\left(1+\tau f_{t}^{t_{i, j}}(r)\right)^{-\frac{\left(t_{i, j}-t\right)}{\tau}}
\end{aligned}
$$

So,

$$
\begin{aligned}
& \frac{\partial u}{\partial r}=-\left(T_{i}-t\right)\left(1+\tau f_{t}^{T_{i}}(r)\right)^{-\frac{\left(T_{i}-t\right)}{\tau}-1} \frac{\partial f_{t}^{T_{i}}(r)}{\partial r} \\
& \frac{\partial v}{\partial r}=\sum_{j=1}^{c} \tau\left(-\left(t_{i, j}-t\right)\right)\left(1+\tau f_{t}^{t_{i, j}}(r)\right)^{-\frac{\left(t_{i, j}-t\right)}{\tau}-1} \frac{\partial f_{t}^{t_{i, j}}(r)}{\partial r}
\end{aligned}
$$


So,

$$
\begin{aligned}
G_{r}(r, t)= & \frac{-\left(T_{i}-t\right)\left(1+\tau f_{t}^{T_{i}}(r)\right)^{-\frac{\left(T_{i}-t\right)}{\tau}-1} \frac{\partial f_{t}^{T_{i}}(r)}{\partial r}}{\sum_{j=1}^{c} \tau\left(1+\tau f_{t}^{t_{i, j}}(r)\right)^{-\frac{\left(t_{i, j}-t\right)}{\tau}}} \\
& +\frac{\left(1+\tau f_{t}^{T_{i}}(r)\right)^{-\frac{\left(T_{i}-t\right)}{\tau}}}{\sum_{j=1}^{c} \tau\left(1+\tau f_{t}^{t_{i, j}}(r)\right)^{-\frac{\left(t_{i, j}-t\right)}{\tau}}} \frac{\sum_{j=1}^{c} \tau\left(t_{i, j}-t\right)\left(1+\tau f_{t}^{t_{i, j}}(r)\right)^{-\frac{\left(t_{i, j}-t\right)}{\tau}-1} \frac{\partial f_{t}^{t_{i, j}}(r)}{\partial r}}{\sum_{j=1}^{c} \tau\left(1+\tau f_{t}^{t_{i, j}}(r)\right)^{-\frac{\left(t_{i, j}-t\right)}{\tau}}}
\end{aligned}
$$

and if we divide each term with $G(r, t)$, we have,

$$
\frac{G_{r}(r, t)}{G(r, t)}=\frac{-\left(T_{i}-t\right)}{1+\tau f_{t}^{T_{i}}(r)} \frac{\partial f_{t}^{T_{i}}}{\partial r}(r)+\frac{\sum_{j=1}^{c}\left(t_{i, j}-t\right)\left(1+\tau f_{t}^{t_{i, j}}(r)\right)^{-\frac{t_{i, j}-t}{\tau}}-1 \frac{\partial f_{t}^{t_{i, j}}}{\partial r}(r)}{\sum_{j=1}^{c}\left(1+\tau f_{t}^{t_{i, j}}(r)\right)^{-\frac{t_{i, j}-t}{\tau}}}
$$

For the partial derivative with respect to $t, G_{t}(r, t)$, we proceed as before,

$$
\begin{aligned}
& \frac{\partial u}{\partial t}=\left(1+\tau f_{t}^{T_{i}}(r)\right)^{-\frac{\left(T_{i}-t\right)}{\tau}}\left[\frac{-\left(T_{i}-t\right)}{1+\tau f_{t}^{T_{i}}(r)} \frac{\partial f_{t}^{T_{i}}}{\partial t}(r)+\frac{1}{\tau} \ln \left(1+\tau f_{t}^{T_{i}}(r)\right)\right] \\
& \frac{\partial v}{\partial t}=\sum_{j=1}^{c} \tau\left(1+\tau f_{t}^{t_{i, j}}(r)\right)^{-\frac{\left(t_{i, j}-t\right)}{\tau}}\left[\frac{1}{\tau} \ln \left(1+\tau f_{t}^{t_{i, j}}(r)\right)-\frac{\left(t_{i, j}-t\right)}{1+\tau f_{t}^{t_{i, j}}(r)} \frac{\partial f_{t}^{t_{i, j}}}{\partial t}(r)\right]
\end{aligned}
$$

So:

$$
\begin{aligned}
G_{t}(r, t)= & \frac{\left(1+\tau f_{t}^{T_{i}}(r)\right)^{-\frac{\left(T_{i}-t\right)}{\tau}}\left[\frac{-\left(T_{i}-t\right)}{1+\tau f_{t}^{T_{i}}(r)} \frac{\partial f_{t}^{T_{i}}}{\partial t}(r)+\frac{1}{\tau} \ln \left(1+\tau f_{t}^{T_{i}}(r)\right)\right]}{\sum_{j=1}^{c} \tau\left(1+\tau f_{t}^{t_{i, j}}(r)\right)^{-\frac{\left(t_{i, j}-t\right)}{\tau}}} \\
& -\frac{\left(1+\tau f_{t}^{T_{i}}(r)\right)^{-\frac{\left(T_{i}-t\right)}{\tau}}}{\sum_{j=1}^{c} \tau\left(1+\tau f_{t}^{t_{i, j}}(r)\right)^{-\frac{\left(t_{i, j}-t\right)}{\tau}}} \\
& \times \frac{\sum_{j=1}^{c} \tau\left(1+\tau f_{t}^{t_{i, j}}(r)\right)^{-\frac{\left(t_{i, j}-t\right)}{\tau}}\left[\frac{1}{\tau} \ln \left(1+\tau f_{t}^{t_{i, j}}(r)\right)-\frac{\left(t_{i, j}-t\right)}{1+\tau f_{t}^{t_{i, j}}(r)} \frac{\partial f_{t}^{t_{i, j}}}{\partial t}(r)\right]}{\sum_{j=1}^{c} \tau\left(1+\tau f_{t}^{t_{i, j}}(r)\right)^{-\frac{\left(t_{i, j}-t\right)}{\tau}}}
\end{aligned}
$$

So, if we divide each term with $G(r, t)$, we have,

$$
\frac{G_{t}(r, t)}{G(r, t)}=\frac{1}{\tau} \ln \left(1+\tau f_{t}^{T_{i}}(r)\right)-\frac{T_{i}-t}{1+\tau f_{t}^{T_{i}}(r)} \frac{\partial f_{t}^{T_{i}}}{\partial t}(r)
$$




$$
-\frac{\sum_{j=1}^{c}\left(1+\tau f_{t}^{t_{i, j}}(r)\right)^{-\frac{\left(t_{i, j}-t\right)}{\tau}}\left[\frac{1}{\tau} \ln \left(1+\tau f_{t}^{t_{i, j}}(r)\right)-\frac{\left(t_{i, j}-t\right)}{1+\tau f_{t}^{t_{i, j}}(r)} \frac{\partial f_{t}^{t_{i, j}}}{\partial t}(r)\right]}{\sum_{j=1}^{c}\left(1+\tau f_{t}^{t_{i, j}}(r)\right)^{-\frac{\left(t_{i, j}-t\right)}{\tau}}}
$$

\section{References}

Ametrano, F., \& Bianchetti, M. (2009). Bootstrapping the illiquidity: Multiple yield curves construction for market coherent forward rates estimation. London: Risk Books, Incisive Media.

Bianchetti, M. (2010). Two curves, one price: Pricing and hedging interest rate derivatives using different yield curves for discounting and forwarding. preprint. Available at SSRN 1334356

Boenkost, W., \& Schmidt, W. (2005). Cross currency swap valuation. Available at SSRN 1375540.

Brigo, D., \& Mercurio, F. (2006). Interest rate models-theory and practice: With smile, inflation and credit. New York: Springer.

Chibane, M., \& Sheldon, G. (2009). Building curves on a good basis. Available at SSRN 1394267

Crépey, S., Grbac, Z., Ngor, N., \& Skovmand, D. (2015). A lévy hjm multiple-curve model with application to cva computation. Quantitative Finance, 15(3), 401-419.

Crépey, S., Grbac, Z., \& Nguyen, H. N. (2012). A multiple-curve hjm model of interbank risk. Mathematics and Financial Economics, 6(3), 155-190.

Cuchiero, C., Fontana, C., \& Gnoatto, A. (2016). A general hjm framework for multiple yield curve modelling. Finance and Stochastics, 20(2), 267-320.

Fanelli, V. (2016). A defaultable HJM modelling of the libor rate for pricing basis swaps after the credit crunch. European Journal of Operational Research, 249(1), 238-244.

Filipović, D., \& Trolle, A. B. (2013). The term structure of interbank risk. Journal of Financial Economics, 109(3), 707-733.

Fujii, M., Shimada, Y., \& Takahashi, A. (2010). On the term structure of interest rates with basis spreads, collateral and multiple currencies. Available at SSRN 1556487

Grbac, Z., Papapantoleon, A., Schoenmakers, J., \& Skovmand, D. (2015). Affine libor models with multiple curves: Theory, examples and calibration. SIAM Journal on Financial Mathematics, 6(1), 984-1025.

Hagan, P. S. (2005). Convexity conundrums: Pricing CMS swaps, caps, and floors. In The best of Wilmott, 305. Chichester: Wiley.

Hagan, P. S., Kumar, D., Lesniewski, A. S., \& Woodward, D. E. (2002). Managing smile risk. Wilmott Magazine, pp. 84-108.

Henrard, M. (2007a). CMS swaps in separable one-factor Gaussian LLM and HJM model. Bank for International Settlements, Working Paper

Henrard, M. (2007b). The irony in the derivatives discounting. Wilmott Magazine, Jul/Aug.

Henrard, M. (2010). The irony in derivatives discounting part II: The crisis. Wilmott Journal, 2(6), 301-316.

Kijima, M., Tanaka, K., \& Wong, T. (2009). A multi-quality model of interest rates. Quantitative Finance, 9(2), 133-145.

Liu, J., Longstaff, F. A., \& Mandell, R. E. (2006). The market price of risk in interest rate swaps: The roles of default and liquidity risks. The Journal of Business, 79(5), 2337-2359.

Lu, Y., \& Neftci, S. (2003). Convexity adjustment and forward libor model: Case of constant maturity swaps. Technical report, FINRISK-Working Paper Series.

Mercurio, F. (2009). Interest rates and the credit crunch: New formulas and market models. Bloomberg Portfolio Research Paper (2010-01).

Mercurio, F. (2010). Libor market models with stochastic basis. Bloomberg Education and Quantitative Research Paper (2010-05).

Mercurio, F., \& Pallavicini, A. (2005). Swaption skews and convexity adjustments. Banca IMI, SSRN Working Paper.

Mercurio, F., \& Pallavicini, A. (2006). Smiling at convexity: Bridging swaption skews and CMS adjustments. Risk August, pp. 64-69.

Moreni, N., \& Pallavicini, A. (2014). Parsimonious HJM modelling for multiple yield curve dynamics. Quantitative Finance, 14(2), 199-210.

Morini, M. (2009). Solving the puzzle in the interest rate market. Available at SSRN 1506046. 
Pallavicini, A., \& Tarenghi, M. (2010). Interest-rate modeling with multiple yield curves. Available at SSRN 1629688.

Pelsser, A. (2003). Mathematical foundation of convexity correction. Quantitative Finance, 3(1), 59-65.

Wu, T. P., \& Chen, S. N. (2010). Modifying the LMM to price constant maturity swaps. Journal of Derivatives, $18(2), 20$.

Zheng, W., \& Kuen Kwok, Y. (2011). Convexity meets replication: Hedging of swap derivatives and annuity options. Journal of Futures Markets, 31(7), 659-678. 